\title{
Altered States: Resting State and Default Mode as Psychopathology
}

\author{
Ben Alderson-Day and Felicity Callard
}

\begin{abstract}
Psychologist Ben Alderson-Day and geographer Felicity Callard share an interest in understanding how interdisciplinary approaches to the brain sciences that involve the social sciences and humanities can help open up new research questions and methods through which to understand pathological and non-pathological states of mind. Both have been interested in the fertility of resting-state research paradigms and the default mode network in this regard. Ben has collaborated on novel experimental investigations of inner experience during the resting state, and Felicity has focused on how tracing historical antecedents of resting-state research might reorient certain current scientific assumptions.
\end{abstract}

Keywords Autism - Default mode network - fMRI - Psychopathology · Psychosis · Resting state

Resting-state functional magnetic resonance imaging research (rsfMRI) investigates spontaneously or intrinsically generated neural activity. While this rapidly expanding field is a recent one - extending back only across the last two decades - attempts to understand what the brain and mind are doing while 'at rest' have a significantly longer history. The human

\footnotetext{
B. Alderson-Day $(\varangle) \cdot$ F. Callard

Durham University, Durham, United Kingdom

e-mail: benjamin.alderson-day@durham.ac.uk; felicity.callard@durham.ac.uk

(C) The Author(s) 2016

F. Callard et al. (eds.), The Restless Compendium, 
sciences have made many attempts to position the human body in particular ways so as to elicit what the mind does when it is not overly preoccupied with responding to external stimuli. In 1930, for example, Hans Berger, in one of his early reports on the use of the electroencephalogram (EEG) in humans, described 'completely relaxed' experimental subjects who 'lay comfortably and with eyes closed on a couch which was insulated from the surrounding by glass feet'. ${ }^{1}$

Resting-state fMRI follows on the heels of such attempts: the 'at rest' condition involves the experimental participant being asked to lie still and relax, either with eyes closed or with eyes open while fixating on a cross. ${ }^{2}$ But this research has also extensively reconfigured assumptions about the working of the brain. In particular, the demonstration that the brain shows a consistent pattern of activation - the 'default mode' - during 'rest' has challenged scientific understandings (from metabolic, cellular and psychological perspectives) about the ways in which a resting body might be accompanied by a distinctly 'restless' brain and mind.

The default mode network (DMN) - a network consistently activated during the 'default mode' - refers to a set of brain regions that tend to show synchronized brain activity when the brain is not engaged in an explicit task. The idea of 'default' derives largely from the observation that these areas (primarily medial prefrontal cortex, posterior cingulate/precuneus, and lateral parietal cortex) tend to deactivate in response to external psychological tasks, indicating 'a heretofore-unrecognized organization within the brain's intrinsic or on-going activity'. ${ }^{3}$ Increased activity of these regions during periods of so-called 'rest' has suggested the importance of introspective processes such as mind wandering, daydreaming and self-reflection. ${ }^{i}$ In this way, the 'default' of the DMN has historically been defined as the flip side of a range of focused, controlled and externally oriented processes: an image in negative of the aware and externally attentive brain. ${ }^{4}$

These attempts to characterize the brain and mind 'at rest' raise complex problems about how one constitutes a 'baseline', and the extent to which a resting state holds consistency in and across individuals across time, space and psychological typology. Additionally, cognitive psychology's significant expertise in cognitive dissection (the careful selection of task and control state which drove many cognitive neuroimaging studies in the 1980s) did not necessarily help in understanding the psychological complexities of an 'uncontrolled' state of rest. But we are now seeing the creative use in

\footnotetext{
i See Chaps. 3, 4 and 5.
} 
cognitive neuroscience of various 'introspective' methods to attempt to capture fleeting moments of consciousness, the emergence of spontaneous thoughts and the moment of transition into a state of mind wandering. ${ }^{5}$ ii

In the remainder of this chapter, we consider some of the difficulties involved in characterizing the resting state in a field that has taken a particular interest in the DMN - namely psychopathology. Psychopathology researchers have increasingly adopted the DMN as a means to investigate clinical symptoms and 'atypical' internal states (see Fig. 2.1).

In part, this explosion of research results from convenience. The DMN can be identified via a resting-state fMRI scan without any task involved: a participant can simply lie in the scanner, and correlated patterns of resting brain activity - functional connectivity - can be found that highlight synchronized 'hubs' of the network. This makes it much easier to acquire data from clinical groups who may struggle with a complex task owing to problems with attention, memory or cognitive control.

Psychopathology researchers have, unsurprisingly, been interested in what the putative functions of the DMN have to tell us about particular disorders. For instance, along with introspective processes, the DMN has been linked to theory-of-mind or 'mentalizing' skills: the ways in which we understand other minds. This has led some to suggest that it may play an important role in autism spectrum disorder, which is historically characterized by problems with understanding others. For instance, an early study by Kennedy and colleagues reported that the DMN 'failed to deactivate' during standard cognitive tasks for a group of autistic adults, which they speculated could reflect 'abnormal internally directed processes at rest'. ${ }^{6}$ Similar observations have been made in research on schizophrenia, although they have mostly been interpreted as reflecting problems with attention and memory. ${ }^{7}$

However, the DMN is just one of many networks that can be observed during a resting-state fMRI scan, and these other resting-state networks (RSNs) are increasingly being used to explore psychopathology. For instance, signals in sensory regions will still tend to be synchronized at rest, which allows for auditory and visual RSNs to be identified. Studying the interaction of these networks with the DMN and other brain regions at rest provides clues as to how sudden sensory experiences - such as hallucinations - can occur spontaneously from brain activity. One current idea is that hallucinations could arise from the contents of an internally

ii See Chap. 6. 


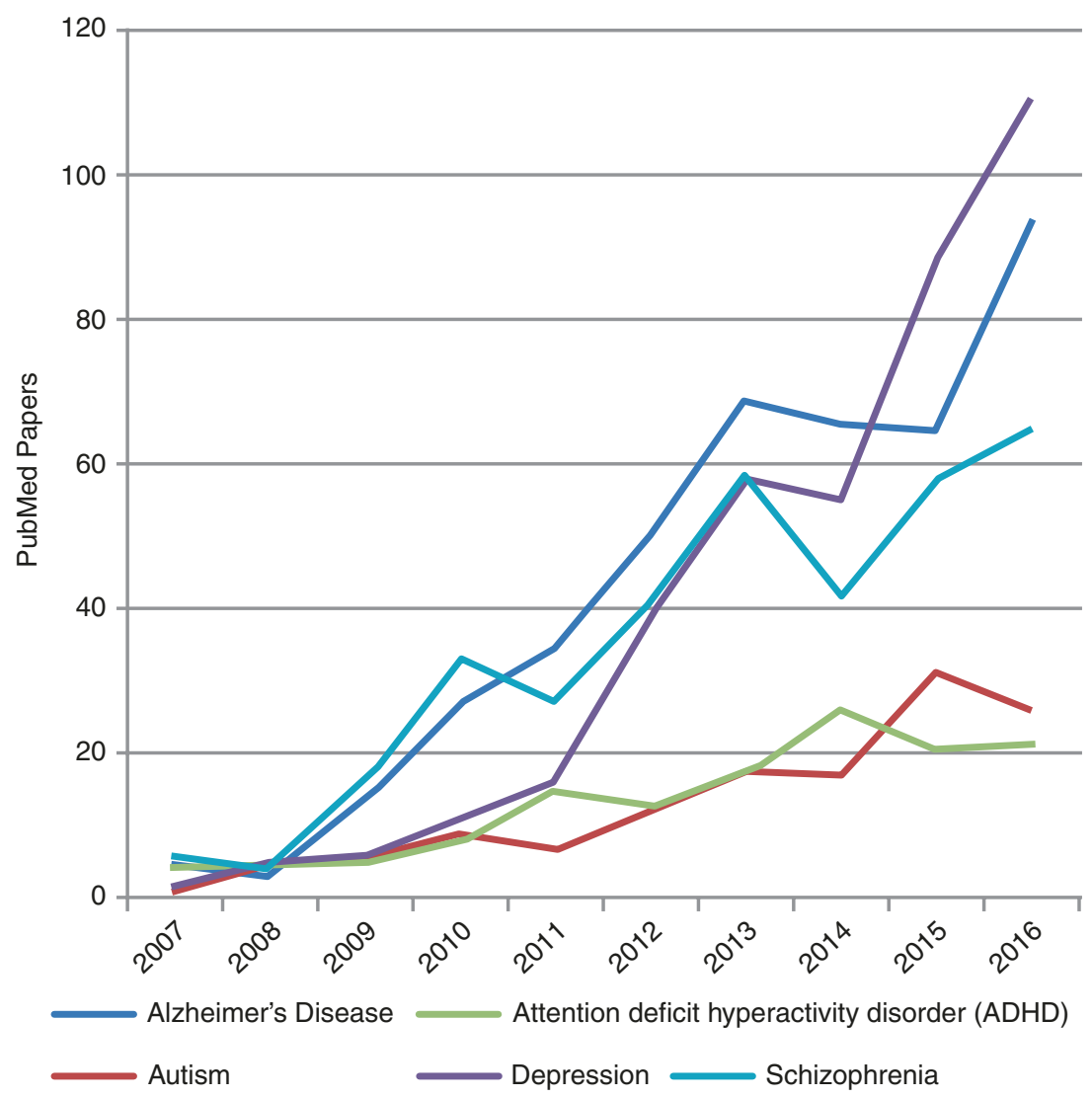

Fig. 2.1 Psychopathology papers published on 'default mode' or 'default network' (PubMed search 18 May 2016; 2016* is a projected estimate for end of year based on papers published to date)

focused state abruptly collapsing into a sensory or perceptual state if the DMN itself is unstable. ${ }^{8}$

Groups of regions related to cognitive control, even if usually associated with an external task rather than rest, also tend to show synchronized activity during a resting-state scan. Along with the DMN, they are also thought to interact with a third set of regions - centred around the insula and anterior cingulate - sometimes called the salience network. This network is involved in identifying significant internal and external changes 
that require a redirecting of attention, and has been proposed as that which controls the switch between the 'internally-focused' states of the DMN and 'externally-focused' states of other networks. This role of this network has attracted considerable interest from researchers who work on psychosis - as many psychotic experiences may seem imbued with strangely significant or meaningful qualities - and also those who research depression and attention deficit hyperactivity disorder (ADHD).

In this way, rest and the resting state have become the window through which many researchers now choose to study specific disorders. There has also arguably been a shift from researchers focusing simply on the DMN to thinking more about how various networks interact with each other over time. This is likely to be particularly important for transient states of mind such as a loss of attention, an intrusive thought or a strange perception. However, there are some important caveats around this work. One concerns methodology, and the other is an interpretative problematic.

First, resting-state scans require participants to keep still. Small head movements over time during a scan can induce systematic biases in the data, and these particularly affect the kinds of statistical analysis required to measure synchronization between brain regions. While certain methods can mitigate such problems, movement during scans has been recognized to be a significant issue, particularly for younger participants. As such, enthusiasm for early findings of connectivity alterations in the DMN in autism, for example, have had to be tempered by concern about the possible effects of participants moving around. ${ }^{9}$

Second, how we should interpret evidence of 'atypical' resting states remains a quandary. Research on different disorders frequently runs in parallel, with limited crosstalk. Very similar findings can therefore end up being interpreted in very different ways: one researcher's 'executive control failure' could be another's 'problem with mentalizing'. In neither case do interpretations become specific explanations.

For the DMN, this may in part reflect its origins in being a 'task-off', 'resting' state. Ever since the DMN emerged, a worry has been that the investigator does not know, ultimately, what is going on in a participant's head: the 'default mode' is a black box that allows for ever-expanding redescription and reinterpretation. When one turns to psychopathology, this problem is magnified tenfold: we know less, not more, about how to interpret the internal states of those with autism, ADHD or psychosis. And how they may differ is not necessarily mysterious: in some cases, mundane contextual factors could have a considerable effect. For example, a resting 
scan will often be acquired after participants have attempted other scans and tasks that are explicitly designed to measure their difficulties or impairments. This could then affect what they are thinking about at rest, in a way that differs from healthy control participants. Being aware of the individual experience of rest is crucial to avoid the possibility of atypicality in results being too rapidly interpreted as pathological.

In this respect, though research on the DMN and rest may be becoming more nuanced, the normative legacy of thinking about the DMN as driving unguided and uncontrolled processes persists in psychopathology research, as it does elsewhere. While the statement 'the resting state is not truly a resting state at all ${ }^{10}$ has now become a shibboleth, it remains far from clear how to parse the complex psychological processes that occur during it. While restingstate fMRI research opens up significant possibilities for understanding the dynamics of altered, unusual and debilitating states of mind, our interpretations must be tempered by a critical view of what counts as a default mode.

\section{Notes}

1. Hans Berger, 'On the Electroencephalogram of Man: Second Report', in Hans Berger on the Electroencephalogram of Man: The Fourteen Original Reports on the Human Electroencephalogram, trans. Pierre Gloor (Amsterdam; New York: Elsevier Pub. Co., 1969), 84, 83. Paper originally published 1930.

2. See for example Rémi Patriat et al., 'The Effect of Resting Condition on Resting-State fMRI Reliability and Consistency: A Comparison between Resting with Eyes Open, Closed, and Fixated', NeuroImage 78 (2013): $463-73$, in which there is explicit attention given to the effects of slightly different 'resting conditions'.

3. Marcus E. Raichle, 'The Brain's Default Mode Network', Annual Review of Neuroscience 38, no. l (2015): 434.

4. Felicity Callard and Daniel S. Margulies, 'What We Talk about When We Talk about the Default Mode Network', Frontiers in Human Neuroscience 8 (2014): 619.

5. For example, see Melissa Ellamil et al., 'Dynamics of Neural Recruitment Surrounding the Spontaneous Arising of Thoughts in Experienced Mindfulness Practitioners', NeuroImage 136 (2016): 186-96; Russell T. Hurlburt et al., 'What Goes on in the Resting-State? A Qualitative Glimpse into RestingState Experience in the Scanner', Frontiers in Psychology: Cognitive Science 6 (2015): 1535.

6. Daniel P. Kennedy, Elizabeth Redcay, and Eric Courchesne, 'Failing to Deactivate: Resting Functional Abnormalities in Autism', Proceedings of the National Academy of Sciences 103, no. 21 (2006): 8275-80. 
7. Susan Whitfield-Gabrieli and Judith M. Ford, 'Default Mode Network Activity and Connectivity in Psychopathology', Annual Review of Clinical Psychology 8 (2012): 49-76.

8. Renaud Jardri et al., 'The Neurodynamic Organization of ModalityDependent Hallucinations', Cerebral Cortex 23, no. 5 (2013): 1108-17.

9. Ralph-Axel Müller et al., 'Underconnected, but How? A Survey of Functional Connectivity MRI Studies in Autism Spectrum Disorders', Cerebral Cortex 21, no. 10 (2011): 2233-43.

10. Abraham Z. Snyder and Marcus E. Raichle, 'A Brief History of the Resting State: The Washington University Perspective', Neuroimage 62, no. 2 (2012): 902-10.

Ben Alderson-Day is a psychologist and cognitive neuroscientist at Durham University. His work has included research on hallucination experiences in clinical and non-clinical populations, and executive function and categorization in autism spectrum disorders.

Felicity Callard is Director of Hubbub and an academic at Durham University (Department of Geography and Centre for Medical Humanities). Her interdisciplinary research focuses on the history and present of psychiatry, psychology, psychoanalysis and the neurosciences. She is co-author of Rethinking Interdisciplinarity across the Social Sciences and Neurosciences (Palgrave Macmillan, 2015).

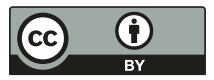

This chapter is distributed under the terms of the Creative Commons Attribution 4.0 International License (http://creativecommons.org/ licenses/by/4.0/), which permits use, duplication, adaptation, distribution and reproduction in any medium or format, as long as you give appropriate credit to the original author(s) and the source, a link is provided to the Creative Commons license and any changes made are indicated.

The images or other third party material in this chapter are included in the work's Creative Commons license, unless indicated otherwise in the credit line; if such material is not included in the work's Creative Commons license and the respective action is not permitted by statutory regulation, users will need to obtain permission from the license holder to duplicate, adapt or reproduce the material. 